Hernández-Prados, M.A., García-Sanz, M. P., Galián-Nicolás, B. \& BelmonteAlmagro, M.L (2019). Implicación de familias y docentes en la formación familiar. Revista Electrónica Interuniversitaria de Formación del Profesorado, 22(3), 61-75.

DOI: http://dx.doi.org/10.6018/reifop.22.3.388971

\title{
Implicación de familias y docentes en la formación familiar
}

\author{
M. Ángeles Hernández Prados, M. ${ }^{a}$ Paz García Sanz, Begoña Galián Nicolás, M. ${ }^{a}$ Luisa \\ Belmonte Almagro
}

Universidad de Murcia

\section{Resumen}

La formación de las familias en España carece de regulación normativa, administrativa e institucional formalmente constituida para tal fin, de modo que la responsabilidad de esta formación se encuentra diluida y depende de los intereses de las AMPA, de los centros educativos, de la Consejería de Educación, de los municipios, y de algunas Organizaciones sin ánimo de lucro. En este artículo presentamos los resultados de la evaluación de necesidades formativas de las familias y los docentes sobre las relaciones familia-escuela en un contexto multicultural, en el que, respondiendo a dos cuestionarios homólogos, han informado un total de 3.639 familias y 225 docentes.

Dichos resultados indican que las familias participan entre poco y algo en actividades de formación dirigidas a ellas y que esta participación desciende a medida que aumenta su nivel de implicación. Los docentes afirman facilitar bastante más la participación familiar en actividades formativas, si bien dicha facilitación también desciende conforme aumenta el grado de implicación. Asimismo, los progenitores del alumnado valoran entre algo y bastante alto otros aspectos de la formación relacionados con la colaboración familiaescuela, incrementándose dicha valoración cuando se trata de los docentes. Por último, más del $80 \%$ de familias y docentes afirma interesarse por recibir formación en lo que respecta a la participación familiar en la educación de los hijos e hijas.

\section{Palabras clave}

Formación; participación; familia; profesorado.

\section{Contacto:}

M. ángeles Hernández Prados, mangeles@um.es, Facultad de educación Universidad de Murcia C.P.30100.

Artículo vinculado al proyecto de investigación I+D+I EDU2016-77035-R, titulado: Compartimos la educación. Programa para el fomento de la participación de las familias en los centros educativos. 


\title{
Involvement of families and teachers in family participation
}

\begin{abstract}
Family training in Spain lacks normative, administrative and institutional regulation, formally constituted for that purpose. As a consequence, responsibility for this purpose is diluted and depends on the interests of the AMPA, educational centres, the Ministry of Education, municipalities and several non-profit organisations. In this article we present the results of the evaluation of the training needs of families and teachers on family-school relationships in a multicultural context, in which, responding to two homologous questionnaires, a total of 3,639 families and 225 teachers have reported information.

These results indicate that families have little participation in training activities for them and that this participation decreases as their level of involvement increases. Teachers affirm that they facilitate family participation in training activities, although such facilitation also decreases as the degree of involvement increases. Also, parents of student's value high or quite other aspects of training related to family-school collaboration, increasing this assessment when it comes to teachers. Finally, more than $80 \%$ of families and teachers affirm that they are interested in receiving training in regard to family participation in the education of their children.
\end{abstract}

\section{Key words}

Training; participation; family; teachers.

\section{Introducción}

La educación de las nuevas generaciones es un reto fascinante y complejo que comparten familias y docentes. Ambos agentes son expertos, cada uno en su ámbito, y se convierten en modelos educativos fundamentales de quienes aprender. De ahí la relevancia de colaborar juntos si quieren que la tarea educativa alcance todo su potencial, especialmente cuando la literatura científica ha dejado constancia de que dicha colaboración contribuye a la mejora del rendimiento y motivación escolar (Fan y Williams, 2010; Jeynes, 2012; Niklas, Cohrssen y Tayler, 2016); reduce el fracaso y abandono académico (Álvarez y Martínez, 2016); mejora el clima del centro y disminuye los problemas de convivencia y acoso escolar (Cross y Barnes, 2014); promueve la democratización y dinamismo de la escuela (Batanova y Loukas, 2014); y previene el consumo de drogas (Roberts, 2018), entre otros.

En España la proliferación y visibilidad de estudios sobre la temática ha contribuido a que la mayoría de centros contemplen entre sus objetivos educativos la mejora de las relaciones colaborativas con las familias. Ahora bien, la ansiada alianza entre familia-escuela no se origina por el simple hecho de ser una afirmación consensuada mundialmente, ni por la lista interminable de beneficios que reporta, pues son varios los trabajos previos que evidencian las dificultades existentes entre ambas instituciones. Al respecto, se contemplan diversas barreras estructurales, organizativas y culturales (Cagigal, 2010); diferentes expectativas (Monceau, 2012); el alejamiento del género masculino (Monceau, 2014); etc.

Todo ello contribuye a evidenciar aún más, la necesidad de formación ante una problemática compleja, que aglutina diversas dimensiones y grados de participación y diferentes agentes educativos. Pero una leve aproximación a los estudios sobre formación en la participación escolar de las familias, nos pone en aviso del tratamiento unilateral de la 
temática, ya que mayoritariamente se ha centrado en el docente, tanto en su formación inicial como en la continua.

Respecto a los futuros docentes, Gomila y Pascual (2015) afirman que la formación académica recibida, caracterizada por la escasez de contenidos curriculares sobre familia, y los periodos de prácticas, en los que se perciben ambos contextos como esferas separadas que deben incluir apoyo, pero no cooperación, constituyen piezas clave en la construcción de representaciones e imágenes, creencias y actitudes hacia la colaboración con las que los alumnos iniciarán su carrera docente. Algunas creencias asentadas en el pensamiento del maestro en formación pueden dificultar la relación familia-escuela, como el hecho de considerar que las familias entorpecen la acción docente, o que solo deben recibir una atención individualizada (Castro, García y Maraver, 2018). De ahí la necesidad de mejorar la formación inicial del profesorado, incrementando en la medida de lo posible la presencialidad de contenidos sobre la relación familia-escuela en el currículum.

No obstante, las diferencias observadas en cuanto a los niveles de participación en función de la etapa (Parra, García-Sanz, Gomariz y Hernández-Prados, 2014) no se deben exclusivamente a parámetros evolutivos y culturales sustentados en la mayor necesidad de la presencia parental a edades tempranas, sino también al modelo de formación inicial que reciben los docentes. En esta dirección, el trabajo de López Larrosa, Richards, Morao y Gómez Soriano (2019) pone de manifiesto que "los profesores y los futuros docentes de educación infantil creían más en la colaboración y menos en la subordinación y la delegación, comparados con los profesores y futuros docentes de educación primaria" (p.59), en consonancia con el trabajo de Vázquez-Huertas y López-Larrosa (2014) que evidencia en los futuros docentes de primaria la asignación de un papel subordinado de las familias en la escuela.

En lo que respecta a los docentes en ejercicio, además de la buena disposición hacia la colaboración, deben desarrollar las habilidades para hacerlo mediante cursos de formación continua, ya que "fijándonos en la forma de funcionar de la cooperación entre padres y profesores en la escuela primaria, identificamos unos mecanismos que pueden parecer contra-intuitivos" (Monceau, 2014, p.72), existiendo el riesgo de "pedagogizar" la relación con las familias al imponer sus propios intereses. Además, los docentes que tienen poco conocimiento de los desafíos y temores de las familias ante la participación en la educación de sus hijos, las juzgan rápidamente de irresponsables (Amatea, Mixon y McCarthy, 2012).

Desde una perspectiva sistémica de la educación, el tratamiento de la participación de las familias en los centros educativos requiere de formación del profesorado y de todas las personas que interactúan con los niños y niñas, especialmente de las familias, y más concretamente, del padre y/o madre. En esta misma línea, Vallespir, Rincón y Morey (2016) proponen un formación bicéfala, en ambos sentidos, en la que las familias reciben una formación planificada, estructurada, implementada, y evaluada contemplada en el ideario del centro educativo, y en el caso de los docentes abarca tanto la formación inicial como continua, y se centra en el siguiente contenido: canales de comunicación, atención individualizada y grupal, tipologías y estrategias de participación, técnicas de dinamización de grupos y educación de adultos.

La formación de las familias en la participación educativa ha sido contemplada por León y Fernández (2018) desde una doble dimensionaliadad: los aspectos conductuales referidos a la educación familiar en el hogar (formación, normas, hábitos y alimentación saludable) y los aspectos académicos referidos a las necesidades educativas de la familia (TIC, técnicas de estudio, relaciones entre iguales, y otros temas concretos). Según Reparaz y Naval (2014), esta formación debe atender a la adquisición de conocimientos, la adopción de 
determinadas actitudes y el desarrollo de diferentes destrezas. No obstante, aunque recientemente se ha producido un aumento del interés por la formación de las familias en este tipo de colaboración, que se encuentra sustentada en las fortalezas educativas que promueve y que ha sido ampliamente avalado por diversas investigaciones, el volumen de experiencias y estudios al respecto no son equiparables a los desarrollados por el colectivo de docentes.

Finalmente, consideramos esencial promover cauces de formación comunitaria conjunta como modelo que favorece y promueve la participación en todas sus modalidades: información, toma de decisiones, planificación, desarrollo y evaluación de la tarea educativa diseñada colaborativamente por familias y docentes. Más específicamente, atiende a los cinco tipos de formación y participación decisiva de las familias en los centros educativos propuestos por Amador y Girbés (2016): informativa, consultativa, decisiva, educativa y evaluadora. No se trata de segregar la formación como dos vías paralelas que nunca llegan a unirse, por el contrario, proponemos un modelo de formación que promueva el encuentro educativo entre docentes y familias durante todo el proceso. Para ello, se generan espacios dialógicos de comunicación positiva desde los que romper los muros y prejuicios que obstaculizan la colaboración, y promover un cambio de mirada de unos a otros que favorezca el reconocimiento y entendimiento mutuo. Destacamos la comunicación como un núcleo esencial en la formación comunitaria familia-escuela, y contemplamos las cuatro recomendaciones transversales señaladas por Macia (2019) para mejorar la comunicación familia-escuela: utilizar los canales de comunicación de forma bidireccional; tratar cuestiones positivas; establecer una rutina comunicativa que aumente la eficacia de la agenda y las circulares; y flexibilizar el horario de las entrevistas individuales y reuniones grupales para facilitar la conciliación. Siguiendo este modelo formativo se plantean los siguientes objetivos de investigación:

1. Conocer la intensidad de cada uno de los niveles de implicación de las familias en las actividades de formación organizadas por el centro, así como la magnitud con la que se dan otros aspectos relacionados con la formación de padres y madres relativa a la educación de los hijos.

2. Identificar en qué grado el profesorado facilita la formación de las familias en cada uno de los niveles de implicación de éstas, así como la intensidad con la que fomenta otros aspectos de la formación de los progenitores del alumnado referida al proceso educativo de los hijos.

3. Analizar la posible existencia de significación estadística, así como el tamaño del efecto, al contrastar el grado de formación de las familias para participar en la educación de los hijos, con la facilitación que los docentes prestan para que se produzca dicha formación.

\section{Metodología}

\section{Participantes}

La investigación se realizó en el municipio de Torre Pacheco (Murcia, España). De una población estimada de 5022 familias de alumnos y alumnas y de 542 docentes de los 14 centros educativos de Educación Infantil, Primaria y Secundaria de dicho municipio, se invitó a participar a todos ellos. Aceptaron la invitación 3639 familias y 225 profesores y profesoras, si bien, una vez depurados los datos, finalmente se contó con una muestra real de 3501 familias. 


\section{Instrumentos}

Para la evaluación de necesidades en la investigación más amplia se utilizaron dos cuestionarios durante la recogida de información:" Valoración de la participación de las familias en la educación de los hijos. Cuestionario a familias" (Hernández-Prados, Gomariz, Parra y García-Sanz, 2017) y "Facilitación de la participación de las familias en la educación de los hijos. Cuestionario a docentes" (Gomariz, Hernández-Prados, García-Sanz y Parra, 2017). En este estudio se recurrió a los 9 ítems de la dimensión de Formación del cuestionario dirigido a las familias y a sus 9 homólogos del cuestionario aplicado al profesorado.

En la Tabla 1 se explicitan los ítems utilizados en la investigación. Se aprecia que del 80 al 83 suponen, en orden ascendente, mayor implicación de los dos colectivos en lo relativo a la formación familiar (conocimiento, asistencia, participación activa e implicación en la gestión de las actividades de formación organizadas por el centro).

Tabla 1.

Ítems de los cuestionarios a familias y docentes utilizados en la investigación

Familias Profesorado

8oF. Tengo información sobre las actividades de formación dirigidas a las familias que organiza el centro educativo.

$80 P$. Procuro que las familias estén informadas de las actividades de formación dirigidas a las mismas que organiza el centro educativo

81F. Asisto a actividades de formación para las familias organizadas desde el centro escolar.
81P. Animo a las familias para que asistan a actividades de formación organizadas desde el centro escolar
82F. Participo activamente en las acciones de formación destinadas a padres y madres (hago preguntas, participo en debates, aplico lo aprendido, etc.).
$82 \mathrm{P}$. Hablo con las familias para que participen activamente en las acciones de formación destinadas a ellas
83F. Me implico en la gestión de actividades de formación para las familias.
83P. Fomento que padres y madres se impliquen en la gestión de actividades de formación para las familias
84F. Necesito formación para mejorar la educación de mi hijo.
84P. Sensibilizo a las familias de la importancia de poseer una adecuada formación en lo que respecta al proceso educativo de sus hijos

85F. La formación ofrecida por el centro contribuye a mejorar las relaciones familia-escuela.

85P. Vinculo la formación ofrecida por el centro con la mejora de las relaciones familia-escuela

86F. La formación de las familias favorece la implicación de los padres/madres en la educación de sus hijos.

86P. Relaciono la formación de las familias con la implicación de éstas en la educación de sus hijos 


\begin{tabular}{ll}
\hline Familias & Profesorado \\
\hline $\begin{array}{l}\text { 87F. El centro facilita la participación } \\
\text { en las actividades formativas } \\
\text { destinadas a las familias. }\end{array}$ & $\begin{array}{l}87 \mathrm{P} \text {. En general, el centro facilita la participación } \\
\text { en las actividades formativas destinadas a las } \\
\text { familias }\end{array}$ \\
\hline $\begin{array}{l}\text { 88F. Estoy interesado en asistir a } \\
\text { actividades de formación para } \\
\text { mejorar mi participación en la } \\
\text { educación de mi hijo. }\end{array}$ & $\begin{array}{l}91 \mathrm{P} \text {. Estoy interesado en asistir a actividades de } \\
\text { formación para mejorar la participación de las } \\
\text { falias en el proceso educativo de sus hijos. }\end{array}$ \\
\hline
\end{tabular}

Los ítems del 80 al 87 se valoraron con una escala ordinal de 5 grados (1-Nunca/nada. 2Pocas veces/poco. 3-Algunas veces/algo. 4-Frecuentemente/bastante. 5-Siempre/mucho), mientras que los ítems $88 \mathrm{~F}$ y 91P se puntuaron mediante una escala dicotómica (Sí/No).

Los dos cuestionarios completos fueron validados por 5 profesores universitarios, así como también por los equipos directivos de los centros participantes y por los directivos de las respectivas Asociaciones de Madres y Padres de Alumnos (AMPA), especialmente en lo que respecta a su contextualización.

Tanto la fiabilidad del cuestionario a familias como la del cuestionario a docentes fueron muy altas (Alfa de Cronbach cuestionario familias=,966; Alfa de Cronbach cuestionario docentes=,981), como también lo fueron las referentes a la dimensión Formación de sendos instrumentos (Alfa de Cronbach Formación cuestionario familias=,839; Alfa de Cronbach Formación cuestionario docentes=,936).

\section{Método y procedimiento}

La investigación realizada es cuantitativa, no experimental, con carácter exploratorio y evaluativa. Concretamente, nos encontramos en la evaluación de necesidades, primera fase del ciclo de intervención socioeducativa (García-Sanz, 2012).

Respecto a la aplicación del cuestionario a familias, tras contactar con los equipos directivos de los centros, los investigadores entregaron personalmente a los mismos los cuestionarios por grupos dentro de sobres con el número exacto de estudiantes, para que dichos equipos los distribuyeran a cada tutor o tutora y fueran éstos/as quienes los confirieran a cada discente para su cumplimentación por parte de sus padres y/o madres. Se tuvo en cuenta el número de estudiantes de habla paterna/materna inglesa o árabe, traduciéndose los cuestionarios a dichos idiomas. Tras cumplirse el plazo establecido, los investigadores se presentaron en los centros para la recopilación de los instrumentos y proceder al análisis de la información.

El cuestionario dirigido al profesorado fue aplicado on-line mediante la plataforma de "Encuestas" de la Universidad de Murcia.

Una vez analizados los datos, se redactaron informes dirigidos al profesorado y a las familias con los resultados más relevantes de la evaluación de necesidades. Estos informes fueron entregados y comentados personalmente por parte de los investigadores a los equipos directivos de los centros y a los directivos de las AMPA para que fueran ellos quienes le dieran la difusión pertinente. 


\section{Técnicas de análisis de datos}

La información recopilada fue analizada con el paquete estadístico SPSS, v. 24. Se utilizó tanto la estadística descriptiva (cálculo de porcentajes, medias y medianas) como la inferencial no paramétrica, ya que se trabajó con escalas ordinales y nominales, además de no cumplirse los principios de normalidad y homocedasticidad. Concretamente, se recurrió a la prueba $U$ de Mann-Whitney, para variables cuasicuantitativas y a la prueba Chi cuadrado para variables dicotómicas. El nivel de significación estadística fue de $\alpha=, 05$.

El tamaño del efecto entre variables medidas en una escala ordinal se realizó mediante el cálculo de la $d$ de Cohen (1988), mientras que entre variables dicotómicas se calculó mediante el coeficiente de contingencia. Cohen (1988) determinó que el tamaño del efecto es suficiente cuando el valor del estadístico $d \geq 5$ y cuando el valor del coeficiente de correlación correspondiente es $r \geq 3$. A dichas puntuaciones el autor mencionado las denominó valores típicos en investigación.

\section{Resultados}

Seguidamente, se muestran los resultados de la investigación, en función de los objetivos planteados en la misma.

\section{Objetivo 1. Formación de familias}

En la Tabla 2 se indican los porcentajes válidos de respuesta de cada una de las categorías de la escala de medida, así como las medias referidas a los ítems que expresan en orden ascendente el grado de implicación de las familias en las actividades de formación organizadas por el centro. En dicha tabla se aprecia que en el ítem $80 \mathrm{~F}$ (conocimiento de las actividades de formación) los porcentajes de respuesta están bastante igualados en las distintas categorías, mientras que en los ítems $81 \mathrm{~F}$ (asistencia a la formación), $82 \mathrm{~F}$ (participación activa en la formación) y $83 \mathrm{~F}$ (implicación en la gestión de la formación), los mayores porcentajes se acumulan en la categoría de nada y los menores en la de mucho. De este modo, excepto en el ítem 8oF, conforme aumenta el nivel de implicación de las familias en su formación, los porcentajes van descendiendo progresivamente al incrementar el grado de la escala.

Del mismo modo, las medias de los ítems expresan claramente su descenso a medida que aumenta el nivel de implicación de las familias en las actividades de formación promovidas por el centro educativo, dirigidas a las mismas (Tabla 2).

Tabla 2.

Porcentajes de respuesta de las familias y medias de los ítems que expresan los niveles de implicación en su formación

\begin{tabular}{lcccccc}
\hline \multirow{2}{*}{ Ítems } & \multicolumn{5}{c}{ Categorías de la escala de medida } & \multirow{2}{*}{ Media } \\
\cline { 2 - 6 } & Nada & Poco & Algo & Bastante & Mucho & \\
\cline { 2 - 6 } & $19,4 \%$ & $19,9 \%$ & $22,4 \%$ & $21,2 \%$ & $17,2 \%$ & 2,97 \\
\hline $80 \mathrm{~F}$ & $33,0 \%$ & $24,6 \%$ & $24,5 \%$ & $11,1 \%$ & $7,2 \%$ & 2,35 \\
\hline $81 \mathrm{~F}$ & $38,3 \%$ & $27,1 \%$ & $20,4 \%$ & $9,3 \%$ & $4,9 \%$ & 2,16 \\
\hline $82 \mathrm{~F}$ & $47,0 \%$ & $25,3 \%$ & $16,7 \%$ & $6,5 \%$ & $4,5 \%$ & 1,96 \\
\hline $83 \mathrm{~F}$ & & & & & & \\
\hline
\end{tabular}


En cuanto a otros aspectos relacionados con la formación de las familias para mejorar la educación de los hijos, en la Tabla 3 se explicitan los porcentajes válidos obtenidos en cada una de las categorías de la escala, así como las medias de los respectivos ítems. Se aprecia que el 30,1\% de las familias reconoce necesitar algo de formación para mejorar la educación de sus hijos (ítem $84 \mathrm{~F}$ ), situándose la media muy cercana a dicha categoría; el 55,2\% opina que la formación ofrecida por el centro contribuye entre algo y bastante a mejorar las relaciones familia-escuela (ítem $85 \mathrm{~F}$ ), ubicándose la media en un valor someramente superior a la categoría de algo ; el $64 \%$ piensa que la formación de las familias favorece entre bastante y mucho la implicación de los padres y madres en la educación de los hijos (ítem $86 \mathrm{~F}$ ), encontrándose en este caso la media cercana a la categoría de bastante ; y el 73,5\% de los progenitores del alumnado percibe que el centro facilita entre las categorías de algo, bastante y mucho las actividades formativas destinadas a las familias, hallándose la media en una puntuación ligeramente superior a la categoría de algo.

Tabla 3.

Porcentajes de respuesta de las familias y medias de los ítems que expresan diferentes aspectos de su formación para mejorar la educación de los hijos

\begin{tabular}{lcccccc}
\hline \multirow{2}{*}{ Ítems } & \multicolumn{5}{c}{ Familias } & \multirow{2}{*}{ Media } \\
\cline { 2 - 6 } & Nada & Poco & Algo & Bastante & Mucho & \\
\cline { 2 - 6 } & $24,7 \%$ & $18,1 \%$ & $30,1 \%$ & $11,7 \%$ & $15,5 \%$ & 2,75 \\
\hline $84 \mathrm{~F}$ & $10,5 \%$ & $13,3 \%$ & $27,4 \%$ & $27,8 \%$ & $21,0 \%$ & 3,35 \\
\hline $85 \mathrm{~F}$ & $7,0 \%$ & $8,4 \%$ & $20,6 \%$ & $31,3 \%$ & $32,7 \%$ & 3,74 \\
\hline $86 \mathrm{~F}$ & $11,5 \%$ & $15,0 \%$ & $24,1 \%$ & $26,5 \%$ & $22,9 \%$ & 3,34 \\
\hline $87 \mathrm{~F}$ & \multicolumn{7}{c}{} & & & & & \\
\hline
\end{tabular}

Respecto a los resultados del ítem $88 \mathrm{~F}$, el $82,7 \%$ de las familias sí está interesado en asistir a actividades de formación para mejorar su participación en la educación de los hijos, mientras que el $17,3 \%$ no lo está.

\section{Objetivo 2. Facilitación docente para la formación de familias}

La Tabla 4 señala los porcentajes válidos de respuesta del profesorado en cada una de las categorías de la escala de medida, así como las medias relativas a los ítems que expresan la facilitación docente de cada uno de los niveles de implicación de las familias en su formación. Se observa que los porcentajes más altos se encuentran en la categoría de bastante, seguida de la de mucho, mientras que los más bajos se ubican en la categoría de nada, seguida de la de poco, en todos los niveles de implicación en la formación familiar. De este modo, el $71,9 \%$ de los docentes procura entre bastante y mucho que las familias estén informadas sobre las actividades de formación organizadas por el centro (ítem 80P); con la misma intensidad, el $67,4 \%$ de los profesores y profesoras anima a los progenitores del alumnado a que asistan a la formación ofertada desde el centro (ítem 81P); también entre bastante y mucho, el $59,8 \%$ del profesorado habla con las familias para que participen en las actividades de formación destinadas a ellas (ítem $82 \mathrm{P}$ ); y el 52,6\% de los docentes, en el mismo grado, sensibiliza a las familias de la importancia de poseer una adecuada formación en lo que respecta al proceso educativo de los hijos (ítem 83P). Por lo tanto, se puede afirmar que el porcentaje de profesores y profesoras que facilita la formación a las familias, disminuye conforme aumenta el nivel de implicación de los padres y madres en su formación. Este hecho también se puede apreciar observando las medias de los ítems, es decir, los docentes fomentan más la participación de las familias en las actividades de 
formación que organiza el centro dirigidas a ellas, cuando dicha participación supone menos implicación para los progenitores del alumnado.

Tabla 4.

Porcentajes de respuesta del profesorado y medias de los ítems que expresan la facilitación docente de los niveles de implicación en la formación de las familias

\begin{tabular}{lcccccc}
\hline \multirow{2}{*}{ Ítems } & \multicolumn{5}{c}{ Profesorado } & \multirow{2}{*}{ Media } \\
\cline { 2 - 6 } & Nada & Poco & Algo & Bastante & Mucho & \\
\cline { 2 - 5 } & $4,9 \%$ & $6,7 \%$ & $16,5 \%$ & $40,6 \%$ & $31,3 \%$ & 3,87 \\
\hline $80 \mathrm{P}$ & $5,8 \%$ & $7,1 \%$ & $19,6 \%$ & $35,3 \%$ & $32,1 \%$ & 3,81 \\
\hline $81 \mathrm{P}$ & $8,0 \%$ & $10,3 \%$ & $21,9 \%$ & $34,4 \%$ & $25,4 \%$ & 3,59 \\
\hline $82 \mathrm{P}$ & $9,8 \%$ & $12,1 \%$ & $24,6 \%$ & $30,4 \%$ & $23,2 \%$ & 3,45 \\
\hline $83 \mathrm{P}$ & & & & & & \\
\hline
\end{tabular}

Respecto al fomento docente de otros aspectos de la formación de los progenitores del alumnado relativa al proceso educativo de los hijos, la Tabla 5 muestra los porcentajes válidos de cada categoría de la escala, así como las medias de los correspondientes ítems. Se puede ver que el $40,9 \%$ del profesorado sensibiliza bastante a las familias de la importancia de poseer una adecuada formación en lo que respecta a la educación de los hijos (ítem 84P); con la misma intensidad, el 36,3\% vincula la formación ofrecida por el centro con la mejora de las relaciones familia-escuela (ítem $85 \mathrm{P}$ ); el 41,1\% de los docentes relaciona bastante la formación de las familias con la implicación de éstas en la educación de los hijos (ítem 86P); y el 40,6\% percibe en el mismo grado, que el centro facilita la participación de las familias en las actividades formativas que ofrece (ítem 837).

En la Tabla 5 también se aprecia que todas las medias se sitúan próximas a la categoría de bastante, luego, en general se puede afirmar que el profesorado facilita con dicha intensidad opiniones positivas en las familias relativas a la formación de éstas, concretamente, las especificadas en el párrafo anterior.

Tabla 5.

Porcentajes de respuesta de los docentes y medias de los ítems que expresan el fomento de diferentes aspectos de la formación familiar para mejorar la educación de los hijos

\begin{tabular}{lcccccc}
\hline \multirow{2}{*}{ Ítems } & \multicolumn{5}{c}{ Profesorado } & \multirow{2}{*}{ Media } \\
\cline { 2 - 6 } & Nada & Poco & Algo & Bastante & Mucho & \\
\cline { 2 - 6 } & $4,4 \%$ & $9,3 \%$ & $17,3 \%$ & $40,9 \%$ & $28,0 \%$ & 3,79 \\
\hline $84 \mathrm{P}$ & $5,4 \%$ & $7,2 \%$ & $21,5 \%$ & $36,3 \%$ & $29,6 \%$ & 3,78 \\
\hline $85 \mathrm{P}$ & $6,7 \%$ & $8,9 \%$ & $16,5 \%$ & $41,1 \%$ & $26,8 \%$ & 3,72 \\
\hline $86 \mathrm{P}$ & $4,5 \%$ & $8,9 \%$ & $16,5 \%$ & $40,6 \%$ & $29,5 \%$ & 3,82 \\
\hline $87 \mathrm{P}$ & & & & & \\
\hline
\end{tabular}

En cuanto a los resultados del ítem 91P, el 80,8\% del profesorado sí está interesado en asistir a actividades de formación para mejorar la participación de las familias en el proceso educativo de sus hijos, mientras que el 19,2\% no muestra interés. 


\section{Objetivo 3. Significación estadística y tamaño del efecto entre familias y docentes}

Al comparar las medias de los ítems contestados por las familias (Tablas 2 y 3 ) con sus homólogos respondidos por el profesorado (Tablas 4 y 5), se observa que, en todos los casos, los docentes perciben facilitar más la actitud positiva e implicación de los padres y madres en las actividades de formación organizadas por el centro que cómo realmente dicen actuar las familias.

Por otra parte, la Tabla 6 señala las medianas de cada uno de los ítems con escala ordinal utilizados en la investigación, respondidos tanto por las familias como por el profesorado. Al igual que ocurre con las medias, se observa que dicho estadístico tiene un valor superior en los ítems cumplimentados por los docentes, excepto en el que hace referencia a la relación que sendos colectivos establecen entre la formación de las familias con la implicación de éstas en la educación de los hijos (ítems 86F y 86P), donde las medianas son iguales.

Tabla 6.

Medianas de los ítems con escala ordinal

\begin{tabular}{lccc}
\hline & Familias & \multicolumn{2}{c}{ Profesorado } \\
\hline Ítems & Medianas & Ítems & Medianas \\
\hline $80 \mathrm{~F}$ & 3 & $80 \mathrm{P}$ & 4 \\
\hline $81 \mathrm{~F}$ & 2 & $81 \mathrm{P}$ & 4 \\
\hline $82 \mathrm{~F}$ & 2 & $82 \mathrm{P}$ & 4 \\
\hline $83 \mathrm{~F}$ & 2 & $83 \mathrm{P}$ & 4 \\
\hline $84 \mathrm{~F}$ & 3 & $84 \mathrm{P}$ & 4 \\
\hline $85 \mathrm{~F}$ & 3 & $85 \mathrm{P}$ & 4 \\
\hline $86 \mathrm{~F}$ & 4 & $86 \mathrm{P}$ & 4 \\
\hline $87 \mathrm{~F}$ & 3 & $87 \mathrm{P}$ & 4 \\
\hline
\end{tabular}

La Tabla 7 indica la significación estadística y el tamaño del efecto al contrastar los ítems dirigidos a las familias con sus homólogos respondidos por los docentes.

Tras el cálculo de la prueba $U$ de Mann-Whitney se demostró que las diferencias entre las medianas de los ítems homólogos son significativas, excepto al contrastar los reactivos $86 \mathrm{~F}$ y 86P. Asimismo, la prueba Chi-cuadrado también arrojó significación estadística al comparar el interés que tienen las familias y los docentes en asistir a actividades de formación para mejorar la participación de padres y madres en el proceso educativo de los hijos (ítems 88F y 91P) (Tabla 7). 
Tabla 7.

Significación estadística y tamaño del efecto

\begin{tabular}{lcc}
\hline $\begin{array}{c}\text { Ítems } \\
\text { contrastados }\end{array}$ & $\begin{array}{c}\text { Significación } \\
\text { estadística }\end{array}$ & $\begin{array}{c}\text { Tamaño del } \\
\text { efecto }\end{array}$ \\
\hline $80 \mathrm{~F}-80 \mathrm{P}$ &, 000 &, 729 \\
\hline $81 \mathrm{~F}-81 \mathrm{P}$ &, 000 & 1,225 \\
\hline $82 \mathrm{~F}-82 \mathrm{P}$ &, 000 & 1,204 \\
\hline $83 \mathrm{~F}-83 \mathrm{P}$ &, 000 & 1,248 \\
\hline $84 \mathrm{~F}-84 \mathrm{P}$ &, 000 & 0,845 \\
\hline $85 \mathrm{~F}-85 \mathrm{P}$ &, 000 & 0,364 \\
\hline $86 \mathrm{~F}-86 \mathrm{P}$ &, 641 & -- \\
\hline $87 \mathrm{~F}-87 \mathrm{P}$ &, 000 & 0,401 \\
\hline $88 \mathrm{~F}-91 \mathrm{P}$ &, 000 & 0,360 \\
\hline
\end{tabular}

Respecto al tamaño del efecto, la $d$ de Cohen indica valores superiores al típico $(d=, 5)$ al contrastar los cinco primeros ítems de sendos colectivos, pero no al comparar los que hacen referencia a si la formación ofrecida por el centro contribuye a mejorar las relaciones familia-escuela (ítems $85 \mathrm{~F}$ y $85 \mathrm{P}$ ) y a si el centro facilita la participación de las familias en las actividades formativas dirigidas a ellas (ítems $87 \mathrm{~F}$ y $87 \mathrm{P}$ ). Por otra parte, el coeficiente de contingencia puso de manifiesto la asociación entre los ítems $88 \mathrm{~F}$ y $91 \mathrm{P}(r \geq 3)$, por lo que el interés por asistir a actividades formativas para mejorar la participación familiar en la educación de los hijos es parecido en familias y docentes.

\section{Discusión y conclusiones}

Nuestros resultados han mostrado, principalmente, que tanto las familias como el profesorado presentan una disposición favorable hacia la formación especializada que les permita mejorar las relaciones de colaboración entre familia-escuela. Esto nos lleva a reafirmar, por un lado, el papel protagonista concedido a la formación como iniciativa para la mejora de la participación (Bernad y Llevot, 2016; Giró y Andrés, 2018), y por otro, la necesidad de incrementar programas de formación (formales o informales), que contemplen la evaluación para comprobar en qué medida contribuyen al cambio (Álvarez Blanco y Martínez-González, 2016; Rivas y Ugarte, 2014; Triana y Rodrigo, 2010).

Sin embargo, el grado de implicación de las familias respecto a la oferta formativa del centro escolar presenta niveles medios bajos, y disminuyen aún más conforme aumenta el grado de responsabilidad y complejidad de la participación, reconociendo estar algo informados, asistir y participar poco, e implicarse muy poco en la gestión de estas actuaciones formativas. Creemos que estar informados es esencial para la colaboración entre ambos agentes (Sarramona y Rodríguez, 2010), pero no es el estado deseable de la participación al que deben aspirar las familias. De hecho, autores como González Falcón 
(2007) consideran que es un paso previo, pero no es participación propiamente dicha. Para que haya cambio el conocimiento "tiene que conectar con las experiencias previas, darle un valor de verdad y percibir que se obtendrá un beneficio por ese cambio" (Martin-Quintana et al, 2009, p.126). De ahí, que resulte esencial la asistencia, participación y colaboración en la formación.

Por otra parte, las familias reconocen que el centro facilita la formación y que ésta contribuye a mejorar las relaciones familia-escuela y la implicación en la educación de los hijos desde el hogar, entendida no solo como atención a los deberes, sino como el desarrollo y ejercicio de la competencia parental, que incluye, según Jiménez e Hidalgo (2017, p.92), "el afecto, el apoyo, la comunicación, la estimulación y la estructuración en rutinas, en el establecimiento de límites, normas y consecuencias, así como en el acompañamiento y la implicación en la vida cotidiana de los hijos e hijas". Pero a pesar de reconocer la importancia de la formación no sienten la necesidad de la misma.

Algunos autores analizan las causas de esta baja implicación y concluyen que no debe imponerse un modelo de formación que responda a los intereses del centro y desatienda los de las familias (Checa y Arjona, 2002) ofreciendo "actividades estandarizadas, sin tener en cuenta las demandas de las personas participantes" (Martínez y Niemelä, 2011, p.71); ni contemplar solo la estructura y cauces de participación, sin promover la motivación de las familias (González Falcón, 2007), pues resultan poco adecuados para la captación de familias. Para motivar la participación de las familias en los programas de formación se debe partir de los intereses de las mismas (estudio de necesidades previas) y promover la motivación extrínseca, intrínseca y trascendente, especialmente esta última, ya que estimula el sentimiento de pertenencia (Lines y Selart, 2013). Además, la formación de familias revierte positivamente en la vida de los participantes, en las relaciones con el centro y con otras familias de diferentes culturas y procedencias (Martínez y Niemelä, 2011).

En lo que respecta a los docentes, coincidimos con lo expuesto por Moriña y Carballo (2018), al sostener que el profesorado reconoce las necesidades formativas y manifiesta el deseo de recibir formación sobre la mejora de la relación con las familias. Los docentes también consideran que el centro facilita la formación de las familias, aunque en mayor medida que las familias, y con bastante frecuencia promueven la importancia de la formación, así como su vinculación con la mejora de las relaciones familia-escuela y la implicación en el hogar. Sin embargo, en su papel como promotores de la formación destinada a las familias, el panorama es muy similar al anterior, ya que facilita con frecuencia información sobre la formación, pero su implicación va disminuyendo progresivamente cuando se trata de promover la asistencia, la participación y la gestión. La negación y negatividad de los profesores ante estas situaciones no es por falta de interés, más bien se trata de la incapacidad que presentan para explorar y potenciar los recursos que tiene la familia, ya que carecen en la mayoría de los casos de la formación necesaria para estimular en los padres y madres las capacidades que tienen en potencia (Galán, 2009).

Para finalizar, consideramos que el modelo de formación también influye en los niveles de implicación, satisfacción y participación (Martin-Quintana et al, 2009), y que el encasillamiento en un modelo académico tradicional de escuela de padres unidireccional centrado en la escucha, se muestra poco recomendable para tratar el tema de la colaboración familia-escuela. Por eso, apostamos por una formación comunitaria conjunta de docentes y familias que colaborativamente se centran en el diseño de proyectos y actuaciones. De este modo, la formación puntual deja paso a un modelo de formación permanente de familias y docentes, que perdura mientras los participantes estén dispuestos a asumir nuevos retos educativos. Asimismo, en consonancia con lo expuesto 
sería pertinente promover la formación de directivos que enfatizara la participación y el liderazgo compartido, ya que estas medidas generan un clima de colaboración sustentado en la corresponsabilidad, así como la adhesión y compromiso con el centro (Rivas y Ugarte, 2014).

\section{Referencias}

Álvarez Blanco, L. y Martínez-González, R. A. (2016). Cooperación entre las familias y los centros escolares como medida preventiva del fracaso y del riesgo de abandono escolar en adolescentes. Revista latinoamericana de educación inclusiva, 10(1), 175-192.

Amador López, J. y Girbés Peco, S. (2016). Formación y participación decisiva de las familias en los centros educativos. Superando las barreras que dificultan la implicación de la comunidad en la escuela. Padres y Maestros, 367, 27-31.

Amatea, E.S., Mixon, K. y McCarthy, S. (2012). Preparing Future Teachers to Collaborate With Families. Contributions of Family Systems Counselors to a Teacher Preparation Program. The Family Journal: Counseling and Therapy for Couples and Families, 21(2), 136-145. doi: https://doi.org/10.1177/1066480712466539

Batanova, M. y Loukas, A. (2014). Unique and interactive effects of empathy, family, and school factors on early adolescents' aggression. Journal of Youth and Adolescence, 43(11), 1.890-1.902.

Bernad Cavero, O. y Llevot Calvet, N. (2016). Las relaciones familia-escuela en la formación inicial del profesorado. Opción, 7, 959-976.

Cagigal, V. (2010). La colaboración educativa familia-escuela. Padres y Maestros, 336, 5-6.

Castro Zubizarreta, A., García-Ruiz, R. y Maraver López, P. (2018). Impacto del practicum en las creencias de los maestros en formación sobre la relación familia-escuela. Revista Brasileira de Educação, 23, 1-19. http://dx.doi.org/10.1590/S1413-24782018230028

Checa, J.C. y Arjona, A. (2002). Actitudes actuales de los jóvenes andaluces hacia los inmigrantes. En L. Serra, (Coord.) Inmigración extranjera en Andalucía (pp. 149-165). II Seminario sobre la Investigación de la Inmigración Extranjera en Andalucía. Andalucía: Junta de Andalucía.

Cohen, J. (1988) (2a . ed.). Statistical Power Analysis for the Behavioral Sciences. New York: Academic Press.

Cross, D. y Barnes, A. (2014). Using Systems Theory to Understand and Respond to Family Influences on Children's Bullying Behavior: Friendly Schools Friendly Families Program. Theory into Practise, 53(4), 293-299.

Fan, W. y Williams, C.M. (2010). The effects of parental involvement on students' academic self-efficacy, engagement and intrinsic motivation. Educational Psychology, 30(1), 5374. doi: $10.1080 / 01443410903353302$

Galán Martín, A. (2009). La influencia del grado de competencia familiar en el desarrollo educativo del niño. En La orientación familiar en el ámbito escolar: la creación de centros de atención a familias en los centros educativos a partir de la experiencia del CAF Padre Piquer (pp. 63-78). Universidad Pontificia Comillas.

García-Sanz, M.P. (2012). Fundamentos teóricos y metodológicos de la evaluación de programas. Murcia: DM. 
Giró Miranda, J. y Andrés Cabello, S. (2018). Profesorado y familias. Actores sin guion. Contextos Educativos. Revista de Educación, 22, 29-44.

Gomariz, M.A., Hernández-Prados, M.A., García-Sanz, M.P. y Parra, J. (2017). Facilitación de la participación de las familias en la educación de los hijos. Cuestionario a docentes. http://hdl.handle.net/10201/53822

Gomila Grau, M.A. y Pascual Barrio, B. (2015). La participación de las familias en el sistema educativo: la percepción del profesorado en formación. Revista Electrónica Interuniversitaria de Formación del Profesorado, 18(3), 99-112.

González Falcón, I. (2007). La participación de las familias inmigrantes en la escuela: necesidades de orientación y formación. XXI: Revista de Educación, 9, 155-169.

Hernández-Prados, M.A., Gomariz, M.A., Parra, J. y García-Sanz, M.P. (2017). Valoración de la participación de las familias en la educación de los hijos. Cuestionario a familias. http://hdl.handle.net/10201/53821

Jeynes, W.H. (2012). A meta-analysis of the efficacy of different types of parental involvement programs for urban students. Urban Education, 47, 706-742.

Jiménez, L. e Hidalgo, M.V. (2017). La incorporación de prácticas basadas en evidencias en el trabajo con familias: los programas de promoción de parentalidad positiva. Apuntes de Psicología, 34(2-3), 91-100.

León Carrascosa, V. y Fernández Díaz, M.J. (2018). Diseño y validación de un instrumento para evaluar la participación de las familias en los centros educativos. REOP-Revista Española de Orientación y Psicopedagogía, 28(3), 115-132.

Lines, R. y Selart, M. (2013). Participation and organizational commitment during change: from utopist to realist perspectives. In H. S. Leonard, R. Lewis, A. M. Freedman, \& J. Passmore (Eds.), The Wiley-Blackwell Handbook of the Psychology of Leadership, Change and Organizational Development (289-311). London: Wiley.

López-Larrosa, S., Richards, A., Morao, S.A. y Gómez Soriano, L.G. (2019). Teachers and trainee teachers' beliefs about family-school relationships. Aula abierta, 48(1), 59-66.

Macia Bordalba, M. (2019). Principales canales para la comunicación familia-escuela: análisis de necesidades y propuestas de mejora. Revista complutense de educación, 30(1), 147165.

Martin-Quintana, J.C. y Máiquez, M.L., Rodrigo-López, M.J., Byme, S., Rodríguez Ruiz, B., y Rodríguez Suárez, G. (2009). Programas de educación parental. Psychosocial Intervention, 18(2), 121-133.

Martínez Gutiérrez, B. y Niemelä, R. (2010). Formas de implicación de las familias y de la comunidad hacia el éxito educativo. Revista Educación y Pedagogía, 56, 69-77.

Monceau, G. (2012). La complexitat de les implicacions dels pares a l'escola o per què la participació dels pares no millora necessàriament els resultats acadèmics dels nens. En Collet; Tort (coord.). Famílies, escola i èxit. Millorar els vincles per millorar els resultats. Barcelona: Fundació Jaume Bofill.

Monceau, G. (2014). Análisis del "alejamiento escolar" de los padres: profundización y ampliación del campo de estudio. Profesorado, Revista de curriculum y formación del profesorado, 18(2), 69-80. 
Moriña, A. y Carballo, R. (2018). Profesorado universitario y educación inclusiva: respondiendo a sus necesidades de formación. Psicología Escolar e Educacional, 22(spe), 87-95.

Niklas, F., Cohrssen, C. y Tayler, C. (2016). Home learning environment and concept formation: A family intervention study with kindergarten children. Early Childhood Education Journal, 44(5), 419-427.

Parra, J., García-Sanz, M.P., Gomariz, M.A. y Hernández-Prados, M.A. (2014). Perfiles de participación de las familias españolas en los centros educativos. En Consejo Escolar del Estado (Ed.). La participación de las familias en la educación escolar (pp. 127-148). Madrid: Ministerio de Educación, Cultura y Deporte.

Reparaz, C. y Naval, C. (2014). Bases conceptuales de la participación de las familias. En Consejo Escolar del Estado (Coord.), La participación de las familias en la educación escolar (pp. 21-34). Madrid: Ministerio de Educación, Cultura y Deporte.

Rivas Borrell, S. y Ugarte Artal, C. (2014). Formación docente y cultura participativa del centro educativo: claves para favorecer la participación familia-escuela. Estudios sobre educación, 27, 153-168.

Roberts, G. (2018). Respuestas del sector de la educación frente al consumo de alcohol, tabaco y drogas (Vol. 10). UNESCO Publishing.

Sarramona López, J. y Rodríguez Neira, T. (2010). Participación y calidad de la educación. Aula abierta, 38(1), 3-14.

Triana B. y Rodrigo M.J. (2010). Modelos y estrategias de intervención ante la diversidad familiar. En: E. Arranz Freijo y A. Oliva Delgado (Coord.), Desarrollo psicológico en las nuevas estructuras familiares (pp. 121-142). Madrid: Pirámide.

Vallespir Soler, J., Rincón i Verdera, J.C. y Morey López, M. (2016). La participación de las familias en el Consejo Escolar y la formación del profesorado. Revista electrónica interuniversitaria de formación del profesorado, 19(1), 31-45.

Vázquez-Huertas, C. y López-Larrosa, S. (2014). Creencias sobre la relación familia-escuela. Cambios en el futuro profesorado tras recibir formación específica. Revista de Estudios e Investigación en Psicología y Educación, 1(2), 111-121. doi: http://dx.doi.org/10.17979/reipe.2014.1.2.35 